\title{
Threshold Size for Ambient Metastability of Rocksalt CdSe Nanocrystals
}

\author{
Keren Jacobs, Juanita Wickham, A. P. Alivisatos* \\ Department of Chemistry, University of California, Berkeley, California 94720 and \\ Materials Science Division, Lawrence Berkeley National Laboratory, University of \\ California, Berkeley, California 94720
}

*Corresponding author. E-mail: alivis@uclink4.berkeley.edu

We show that metastable rocksalt CdSe nanocrystals can persist at ambient pressure depending on the physical size of the particle. The size-dependence of the hysteresis loop was measured for the solid-solid transition in CdSe nanocrystals, between four- and sixcoordinate structures. A systematic shift of the entire hysteresis loop to lower pressure results in a threshold size of $\sim 11 \mathrm{~nm}$ for ambient metastability of the six-coordinate rocksalt structure. Smaller nanocrystals transform back to the tetrahedrally bonded structure as occurs in the CdSe bulk solid. Surface energy contributions are used to explain the shift. The results have important implications for the optimum synthesis of metastable nanocrystal solids under ambient conditions. 
Many solids transform to denser structures under applied pressure, resulting in materials with novel properties without any change in the material composition. Highpressure phases can persist in a metastable state at ambient pressure if sufficiently large energetic barriers hinder transformation to the more energetically favorable structure. The best known example is diamond, which is a high-energy structure of carbon relative to graphite. Researchers have recently succeeded in trapping the high-pressure nonmolecular phase of nitrogen, a very dense material that could have practical use. ${ }^{1}$ Metastable solids that are otherwise unavailable in the bulk solid have been engineered in nanoscale materials through complicated synthetic strategies. Some examples include the rocksalt phase of $\mathrm{CdS}$ for $100 \mathrm{~nm}$ particles synthesized in polymer films in the presence of certain surfactants and the rocksalt phase of CdSe trapped as thin layers electrodeposited on a gold substrate..$^{2,3}$

Despite interest in materials on the nanoscale, the influence of physical size on structural metastability is not well understood. In comparison, thermodynamic influences are well documented in the literature, such as in the synthesis of nanocrystalline alumina systems. ${ }^{4}$ The observation of variable thermodynamic stability in alumina systems on particle size was explained by the notion that smaller crystallites preferentially favor polymorphs with surfaces of relatively low energy. This translates in macroscopic terms to the idea that the most efficient way to pack oranges in a crate may differ if a small refrigerator drawer suddenly binds the packing container. The influence of physical size is also known in melting studies on a wide variety of nanocrystal materials, for which a depression in melting temperature is observed with decreasing size. ${ }^{5-7}$ The liquid phase is 
stabilized relative to the solid in small sizes because surface energy contributions are minimized in liquids.

The influence of size on metastability is comparatively more complicated to understand because thermodynamic effects can be convoluted in these systems with kinetics effects. Metastable systems here imply the persistence of the state over "long" time scales, days or years, at room temperature. Theoretical estimates for lifetimes of metastable states can be off by many orders of magnitude because they must include largely unknown details of microscopic transition pathways. ${ }^{8,9}$ The existence of a threshold size for ambient structural metastability was previously predicted from presumed increase of the transformation barrier with size ${ }^{10}$ or alternatively based on novel crystal shapes with desirable surface energies. ${ }^{11}$ A threshold size in the CdSe nanocrystal system is experimentally demonstrated here, showing that thermodynamic surface effects dominate the size-dependent behavior of the structural transformation.

The CdSe nanocrystal system has been used as a model for structural studies. ${ }^{12,13}$ The nanocrystals undergo the same structural transition as in corresponding bulk, but are synthesized as nearly monodisperse nearly defect-free crystals with controlled shape. ${ }^{14}$ The thermodynamic phase is a tetrahedrally bonded wurtzite/zinc blend structure at atmospheric pressure. The crystal transforms under pressure to a more densely packed six-coordinate rocksalt structure with an $18 \%$ reduction in volume (Fig. 1). This transformation exhibits hysteretic behavior characterized by a large difference in pressure required to induce the forward compared to the reverse transition (Fig. 2 inset). A previous study reported nearly constant hysteresis widths of $\sim 6 \mathrm{GPa}$ for $\mathrm{CdSe}$ nanocrystals $3-13 \mathrm{~nm}$ in diameter. ${ }^{12}$ This massive hysteresis compared to the $\sim 2 \mathrm{GPa}$ 
width in CdSe bulk ${ }^{15}$ is attributed to the large barriers associated with the single-domain behavior of the transformation. In bulk $\mathrm{CdSe}$, the tetrahedrally bonded structure recovers with the release of pressure. ${ }^{15}$

Nearly spherical nanocrystals between 3-13 nm in diameter with narrow size distribution $(\sigma=5-10 \%)$ were synthesized using known methods. ${ }^{16,17}$ Sizes were determined based on transmission electron micrograph (TEM) images. The nanocrystals were coated with an organic ligand (tri-octyl phosphine oxide) and dissolved in ethylcyclohexane, which acts as both a solvent and a pressure-transmitting medium. Pressures between 0-10 GPa were applied using a diamond anvil pressure cell (DAC), and measured with standard ruby florescence techniques. ${ }^{18}$ The transition was monitored through changes in the visible absorption of the sample because the tetrahedrally bonded structure is a direct band-gap semiconductor and has an electronic absorbance peak in the visible, whereas the six-coordinate structure is an indirect band gap semiconductor with a featureless absorption spectrum. ${ }^{13}$

The reverse transition pressure from the rocksalt to the tetrahedrally bonded structure is shown as a function of nanocrystal size in Figure 2. The transition pressure shifts to lower pressure with increasing size and crosses to a regime of metastability at $\sim 10 \mathrm{~nm}$, where the reverse transition pressure is less than 1 atm ( 0 GPa on the scale). Xray diffraction $(\mathrm{XRD})$ measurements were collected for an $11 \pm 1 \mathrm{~nm}$ sample. The sample was pressurized to $9 \mathrm{GPa}$ to induce transformation to the rocksalt phase. The pressure was subsequently released to form air pockets (i.e. atmosphere) and reapplied to <1 GPa to prevent sample leakage during data collection. Characteristic rocksalt peaks are present in the corresponding XRD pattern, in addition to peaks characteristic of the recovered 
tetrahedrally bonded phase (Fig. 3). The metastable rocksalt phase comprises $\sim 20 \%$ of the sample at ambient pressure, as calculated from structure factors for the respective reflection planes. An additional pattern obtained on residual sample (remaining on a ruby chip placed for pressure calibration) after opening the DAC also contains the characteristic rocksalt peaks.

A threshold size of $\sim 11 \mathrm{~nm}$ based on the XRD measurements is slightly higher than the $10 \mathrm{~nm}$ size indicated by the optical data. This can be attributed to the transformation kinetics because the optical data was collected in three-minute intervals, whereas the XRD patterns were collected over the course of days. Longer waiting times increase the probability that the reverse transformation takes place, resulting in larger threshold sizes. Nevertheless, the size-dependence of the reverse transition pressure is extremely strong, suggesting that the crossover size is "sharp" and particles above the threshold size should exhibit metastable lifetimes much longer than just days. The difference in threshold size could alternatively be an artifact- a consequence of lower sensitivity in the optical measurements, as this technique is an indirect indicator of the structures. If in fact the transformation kinetics near the threshold size is considerable, the kinetics may also contribute to the presence of both recovered and metastable phases in the XRD pattern (Fig. 3).

The primary reason for the mixture of recovered and metastable phases is likely due to the size distribution in the sample, considering the strong dependence of the reverse transition pressure on crystal volume (Fig. 2). The relatively narrow $10 \%$ distribution in the $11 \mathrm{~nm}$ sample diameter corresponds to as much as $30 \%$ distribution in the volume. To explore this issue, the particle sizes in the $11 \mathrm{~nm}$ sample were extracted 
from the Debye-Scherrer broadening of the peaks in the XRD patterns. The diffraction peak widths indicate a structural coherence length in the $11 \mathrm{~nm}$ particles equivalent to an average of $\sim 10 \mathrm{~nm}$ in diameter at ambient pressure. The peak widths for the recovered tetrahedrally bonded structure is equivalent to an average $\sim 8 \mathrm{~nm}$ in diameter, and the metastable rocksalt domain to an average $\sim 7.7 \mathrm{~nm}$ in diameter. The $7.7 \mathrm{~nm}$ is consistent with an $18 \%$ volume reduction accompanying the structural transformation from the original $10 \mathrm{~nm}$ simulated diameter tetrahdrally bonded phase. The $8 \mathrm{~nm}$ size for the recovered tetrahedrally bonded fraction of the sample, compared to the $10 \mathrm{~nm}$ simulated for the entire sample, supports the explanation that the mixture of phases in Figure 3 is largely due to the size distribution of the sample.

An alternative, but unlikely, explanation for the mixture is the coexistance of both phases within individual nanocrystals. Although multiple structures can be transiently present in a bulk solid, the interface is unstable and should grow quickly across a nanocrystal. Significant broadening of the XRD peaks to an estimated 0.4 FWHM in Figure 3 would result if $20 \%$ of each nanocrystal was in the rocksalt phase. Instead, the experimental peak width corresponds to a domain size of $\sim 7.7 \mathrm{~nm}$ in diameter, which is consistent with the notion that each nanocrystal must be in either one structure or the other. It is believed that like the $8 \mathrm{~nm}$ particles previously shown to be single-domain after a transformation cycle, ${ }^{12}$ the metastable particles observed here are single domains of the rocksalt structure.

Figure 2 shows the empirical source of the threshold size is a systematic lowering of the reverse transition pressure with size. We emphasize that this trend is directly correlated to a relative shift of the entire hysteresis loop, because the hysteresis width is 
nearly size-insensitive over this size range. ${ }^{12}$ A similar shift was previously observed for 2-4 $\mathrm{nm}$ particles, and is now observed over a significantly extended size range. The shift reflects relative changes of the thermodynamic transition pressure with nanocrystal size, if it is assumed that the location of this pressure within the hysteresis loop does not significantly vary with size. The validity of this assumption is not currently known, although it is established that the thermodynamic pressure is unlikely to be precisely centered within the hysteresis loop. ${ }^{12}$ The reasoning is that the surface energies are a significant contribution to the total free energy of the nanocrystal and these energies are different in the two structural phases. With decreasing crystal size, the thermodynamic transition pressure shifts to favor the structure with the lower surface energy. The rocksalt structure of CdSe nanocrystals is thought to have higher surface energy than the tetrahedrally bonded phase because the shape change accompanying the transformation alters the crystallographic facets normally exposed in an annealed particle. The known shape change for the CdSe nanocrystals indicates these high-energy faces are predominantly the (111) faces parallel to the crystallographic c axis (Fig. 1). In this respect and based on the given shape change, rods grown along c-axis are less desirable for producing metastable materials due to relatively large (111) surface areas exposed, such that the threshold size should also depend on crystal shape. In the limit of a bulk solid, the rocksalt structure is not metastable despite minimal influence of the surface, because the hysteresis width is too narrow. The threshold size is thus a trade-off between the thermodynamics favoring larger systems, and massive hysteresis occurring in the nanocrystal system. This work is further illustration that surface energy can play a 
dominant role in determining the relative stability of structural nanocrystalline phases, and must be taken into consideration in studies of nanocrystal structural phase changes. In contrast, the prediction for structural metastability in large nanocrystal sizes based on kinetics effects is unsuccessful for this system. This prediction was based on comparisons to magnetism in nanoparticles, used in everyday storage media. Magnetic and structural transitions are analogous in thermodynamics and statistical mechanics, and their behaviors in nanoparticles are often compared for general insight into transitions in small systems. Magnetic transitions between up and down magnetic moments produce a metastable state in which the material remains magnetized (remanent magnetization) after the removal of an applied magnetic field. The barrier to reversing the magnetization strongly increases with particle size in nanocrystals, because magnetic reversal involves synchronized coordination of the entire nanocrystal solid. The result is a superparamagnetic limit, $\sim 10 \mathrm{~nm}$ in Fe:Co alloys, ${ }^{19}$ which is the minimum threshold size for observable remanent magnetization. Kinetic barriers to the solid-solid transition were previously presumed to similarly increase with nanocrystal size, from which ambient structural metastability in CdSe nanocrystals would be produced as the hysteresis width broadened. However, nanocrystals were recently found to transform between solids through nucleation mechanisms and the hysteresis width does not broaden with size. ${ }^{12}$ As a consequence, the thermodynamics shift rather than a size-dependence of the kinetics is responsible for the structural metastability. Although production of metastable phases in both these nanocrystalline transitions depends on the physical size of the particle, the engineering approaches for producing metastability are now known to be quite different in the two cases. 

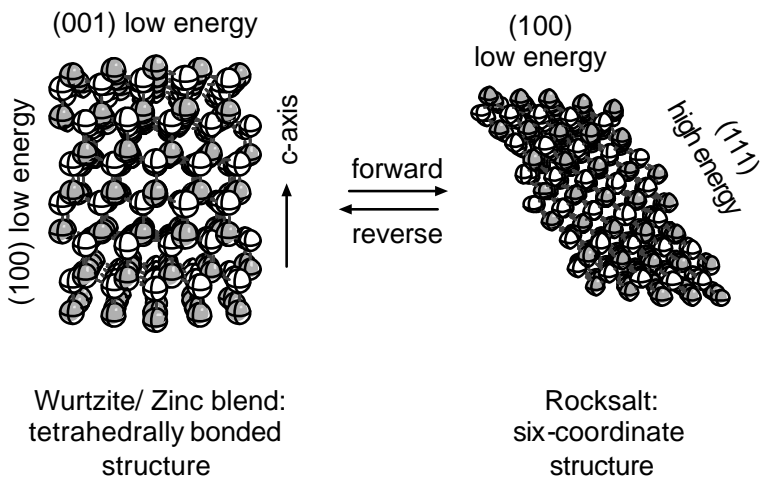

Figure 1. A schematic of the shape change in nanocrystals accompanying the CdSe solidsolid transformation, between four-and six-coordinate structures ${ }^{20}$ Crystallographic indexes of several exposed faces are labeled. The shape change exposes high-energy rocksalt faces, such as the (111) face, that would otherwise not be exposed in an annealed particle. The shape change takes place because the transition is a single-domain process and room temperature is too low for surface rearrangement to occur, as it is below the $575 \mathrm{~K}$ limit at which interparticle diffusion occurs and the crystals begin to aggregate. ${ }^{21}$ The surface energies can be a significant contribution to the total free energy of the nanocrystal since considering that over one third of the atoms are on the surface in $3 \mathrm{~nm}$ particles. 


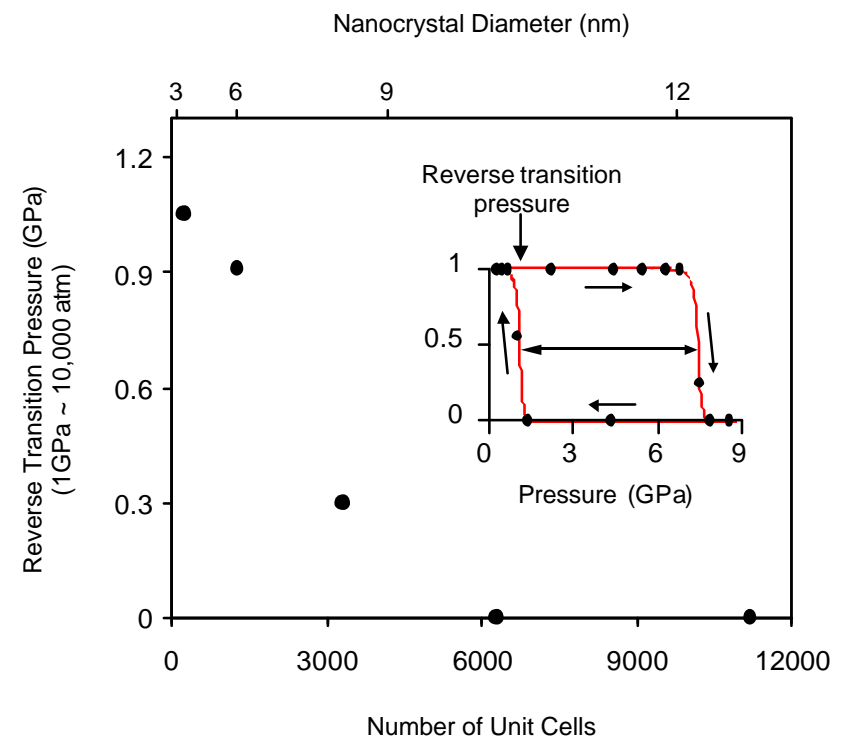

Figure 2. Reverse transition pressure versus size at room temperature in $\mathrm{CdSe}$ nanocrystals at room temperature. The inset is a sample hysteresis loop for $3.5 \mathrm{~nm}$ diameter nanocrystals, from which the reverse transition pressure is $50 \%$ transformation back to the tetrahedrally bonded structure. The loop starts at low pressure and proceeds in the direction of the arrows, as the normalized ratio of sample transformed is monitored with optical measurements. Sizes with reverse transitions less than ambient pressure are trapped in the metastable rocksalt structure. 


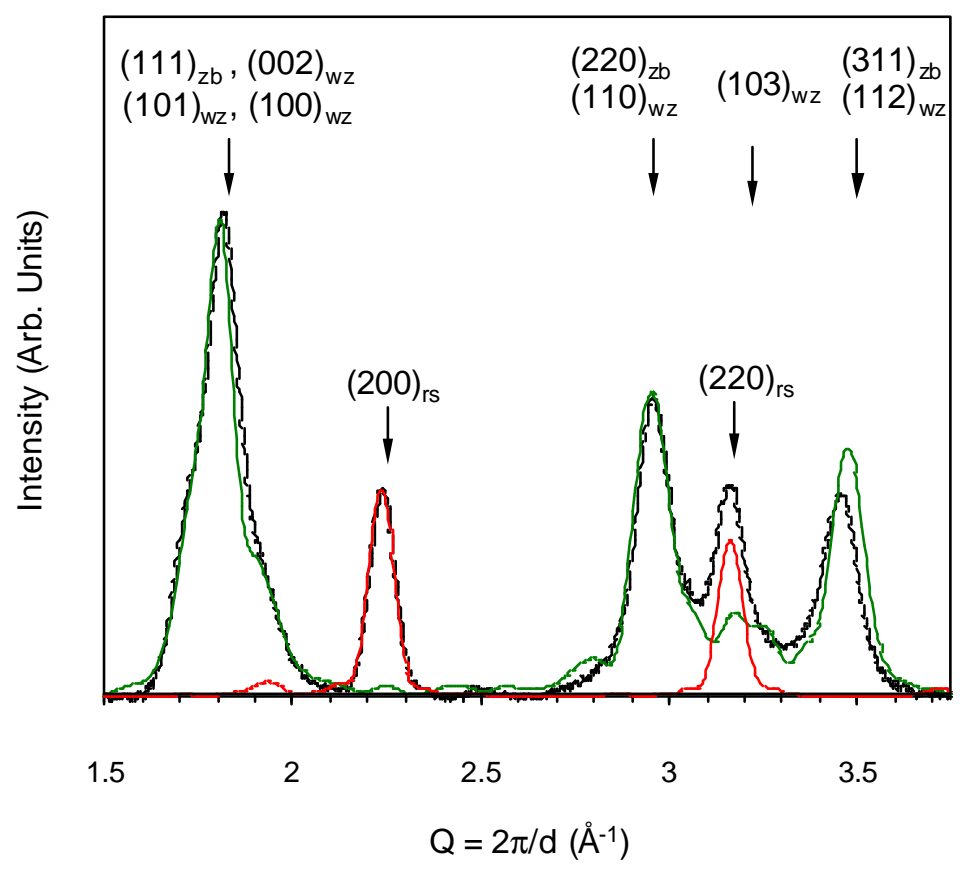

Figure 3. Powder x-ray diffraction (XRD) pattern of $11 \mathrm{~nm}$ CdSe nanocrystals after a transition cycle and the release of pressure at room temperature. The crystallographic indexes are assigned to the respective features. The figure shows the presence of both recovered tetrahedrally bonded wurtzite/zinc-blend crystals and metastable six-coordinate rock salt crystals. The (200) rocksalt peak is exclusive to the rocksalt structure, while the (220) peak overlaps with a potential (103) peak of the tetrahedrally bonded structure. The red line is the simulated contribution of the rocksalt structure, the green line for the tetrahdrally bonded structure. This pattern was collected at the Stanford Synchrotron Radiation Laboratory (SSRL), Menlo Park, California on Beamline 10-2 with photon energy of $17 \mathrm{KeV}$. 
Acknowledgement:

We thank S. Prilliman and D. Zaziski for technical assistance; D. Near and the staff of SSRL for assistance with the synchrotron data collection; X. Peng for help with the synthesis. This work was supported by the Director, Office of Energy Research, Office of Basic Energy Sciences, Division of Materials Sciences, of the U.S. Department of Energy under Contract No. DE-AC03-76SF00099. X-ray diffraction experiments were performed using the facilities of the University of California-LLNL PRT at the Stanford Synchrotron Radiation Laboratory, which is operated by the DOE, Division of Chemical Sciences.

References and Notes

(1) Eremets, M. L.; Hemley, R. J.; Mao, H.; Gregoryanz, E. Nature 2001, 411, 170.

(2) Lin, J.; Cates, E.; Bianconi, P. A. J. Am. Chem. Soc. 1994, 116, 4738.

(3) Zhang, Y.; Hodes, G.; Rubinstein, I.; Grunbaum, E.; Nayak, R. R.; Hutchison, J. L. Adv. Mat. 1999, 11, 1437.

(4) McHale, J. M.; Auroux, A.; Perrotta, A. J.; Navrotsky, A. Science 1997, 277, 788 .

(5) Goldstein, A. N.; Echer, C. M.; Alivisatos, A. P. Science 1992, 256, 1425.

(6) Coombes, C. J. J. Phys. F 1972, 2, 441. 
(7) Buffat, P.; Borel, J. P. Phys. Rev. A 1976, 13, 2287.

(8) Martonak, R.; Molteni, C.; Parrinello, M. Comput. Mater. Sci. 2001, 20, 293.

(9) Shimojo, F.; Ebbsjö, I.; Kalia, R. K.; Nakano, A.; Rino, J. P.; Vashishta, P. Phys. Rev. Lett. 2000, 84, 3338.

(10) Chen, C. C.; Herhold, A. B.; Johnson, C. S.; Alivisatos, A. P. Science 1997, 276, 398.

(11) Brus, L. E.; Harkless, J. A. W.; Stillinger, F. H. J. Am. Chem. Soc. 1996, $118,4834$.

(12) Jacobs, K.; Zaziski, D.; Scher, E. C.; Herhold, A. B.; Alivisatos, A. P. Science 2001, 293, 1803.

(13) Tolbert, S. H.; Herhold, A. B.; Johnson, C. S.; Alivisatos, A. P. Phys. Rev. Lett. 1994, 73, 3266.

(14) Peng, X.; Manna, L.; Yang, W.; Wickham, J.; Scher, E.; Kadavanich, A.; Alivisatos, A. P. Nature 2000, 404, 59.

(15) Yu, W. C.; Gielisse, P. J. Mater. Res. Bull. 1971, 6, 621-\&.

(16) Murray, C. B.; Norris, D. J.; Bawendi, M. G. J. Am. Chem. Soc. 1993, 115, 8706.

(17) Peng, X. G.; Wickham, J.; Alivisatos, A. P. J. Am. Chem. Soc. 1998, 120, 5343.

(18) Barnett, J. D.; Block, S.; Piermari, G. J. Rev. Sci. Instrum. 1973, 44, 1-9.

(19) Richter, H. J. J. Phys. D-Appl. Phys. 1999, 32, R147. 
(20) Wickham, J. N.; Herhold, A. B.; Alivisatos, A. P. Phys. Rev. Lett. 2000, 84,4515 .

(21) Herhold, A. B. Kinetics of Solid-Solid Phase Transitions in Semiconductor Nanocrystals, University of California, Berkeley, 1997. 